\title{
SIMULEM: Introducing Goal Oriented Behaviours in Crowd Simulation
}

\author{
Sébastien Paris ${ }^{1}$, Delphine Lefebvre ${ }^{2}$, and Stéphane Donikian ${ }^{2}$ \\ 1 Cognitive Science Lab. - Laval University \\ Dep. of Computer Science and Software Engineering \\ Pavillon Pouliot, 1065 rue de la Médecine - G1K 7P4 Quebec, QC - Canada \\ parissebastien@free.fr \\ 2 Bunraku team - IRISA \\ Campus de Beaulieu - 35042 Rennes cedex - France \\ dlefebvr@irisa.fr-donikian@irisa.fr
}

Summary. Short description of the paper...

\section{Introduction}

Architectural domain needs for crowd simulation in order to validate the design of buildings that can host a large number of users, such as train stations, bus stations, or airports. Some simulation tools already exist to study flows of people inside constrained areas, but they only address the most visible behaviour of human beings, the navigation. Then, the environment is only considered as a set of obstacles to avoid, and the objectives of the simulated pedestrians are only a set of predefined destinations in the environment. However, real humans achieve higher level goals usually related to the interactions they must accomplish within their environment, such as in a train station: buying a ticket, consulting the departure board, etc. These interactions have a strong impact on the navigation tasks because they define a set of emergent destinations for a simulated pedestrian, and also provoke waiting queue formations. Thus, a goal oriented behaviour based on interactions with equipments should be placed at the heart of a simulation tool in order to study the everyday usage of public buildings.

We propose in this paper a new crowd simulation architecture which manages this kind of high level behaviours for virtual humans. Our approach combines two main components. First, a realistic environment description represents exactly the geometry and topology of a public building. This environment is informed with interactive 
objects which situate the interaction opportunities, allowing to include them in the decision process associated with the navigation task of a virtual human. Second, our virtual human is able to take advantage of this informed environment to perform some interactions with regard to its goal oriented behaviour. Thus, the decision process of our virtual human takes into account its final goal, including all the necessary interactions to reach this goal and their impact on the navigation.

This paper is organized as follows. First, section 2 exposes a state of the art about crowd simulation. Following, section 3 describes our agents' goal oriented behavioural model. Then, we present in section 4 an overview of our simulation architecture, with the different phases of a simulation. Finally, we demonstrate our approach over examples in section 5 . We conclude by presenting our perspectives in section 6 .

\section{Related work}

- Helbing [1][2][3]

- Crowd Dynamics / GK Still [4]

- Legion Limited [5]

- Crowd [5]

\section{Goal oriented behavioural model}

\subsection{Model overview}

We have ground our behavioural model on a cognitive approach by adopting the perception - decision - action loop. The decision process of our autonomous agent is based on a multilayer model, which can easily be compared to A. Newell's pyramid [6] (Figure 1 $(a)$ ). Each layer in this pyramid is independent from the others, only requiring a set of identified data to work. One can notice that only the upper layer of the pyramid is not represented in our model. This layer corresponds to the social behaviours, which are not directly addressed by this work.

Let us introduce the other layers of the pyramid, and the way they communicate (Figure 1(b)):

Rational: this layer is in charge of the logical decisions of the autonomous agent. These decisions are based on un-embodied notions describing the goals and subgoals of the agent at a conceptual level. This layer also organises these goals to find the feasible actions independently of their location. Finally, this layer performs a pre-selection over the agent's goals, to reduce their number for the next decision phase. 


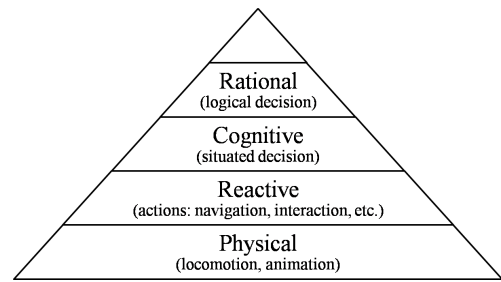

(a) Overview of the decision mechanisms of an agent.

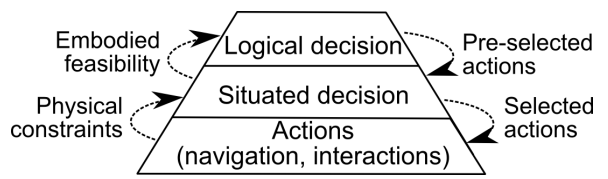

(b) Communication between the three upper layers of the pyramid.

Fig. 1: Our multilayered model of decision in the same way as Newell's pyramid.

Cognitive: this layer is in charge of the situated decision of the autonomous agent. This decision refines the pre-selection made at a rational level by taking into account the place where actions can be performed. Thereby, the impact of the actions on the necessary movement of the autonomous agent is considered. Finally, this layer elects one primary goal to perform, and a set of secondary goals. The remaining goals, if any, are temporarily ignored by the agent. The primary goal is managed by a behaviour which has a full control over the agent's reactive abilities, such as the navigation. The secondary goals are managed by concurrent behaviours, which only have a limited control over the agent's reactive abilities, in order to influence them. A typical control is to divert the agent from an optimal trajectory towards its primary goal in order to perform a secondary goal while walking. For example, an agent can read a sign while going to a cash dispenser, and so slow down to have enough time to fulfill the interaction.

Reactive: this layer is designated for the management of the finally selected actions. The short term decisions made at this stage only manage physical behaviours, like the navigation [7] or the perception [8] of the autonomous agent, but also the actions on objects. Finally, this layer directly control the lower layer of the pyramid by producing low level actions.

Physical: this layer is in charge of the agent's physiological management. In our case, this management is limited to the locomotion and optionally the animation of the agent. Both of these tasks are a direct application of the MKM model [9] developed in our team.

We must emphasise that this goal oriented decision management needs an informed environment description to resolve the goals selection. Indeed, the rational layer must manipulate a conceptual representation of the environment in order to perform the pre-selection, and then the cognitive part requires to situate these concepts in order to That is why we propose an architecture to describe and situate interaction concepts. 


\subsection{Interaction concepts}

We propose in our model a generic architecture defining the way interaction concepts operate together: $\mathrm{BIIO}$ [10] for Behavioural Interactive and Introspective $\mathrm{Ob}$ jects. Indeed, $B I I O$ proposes an operational framework, which can be extended by specialising or unifying the proposed high level concepts. These concepts are mainly oriented towards interaction, which is certainly the kind of behaviours which have the most impact on the environment, and so on the results of a crowd simulation. We define an interaction to be any action between an autonomous agent and another embodied element situated in the environment, such as another autonomous agent or an equipment. The set of concepts we introduce are defined in figure 2 .

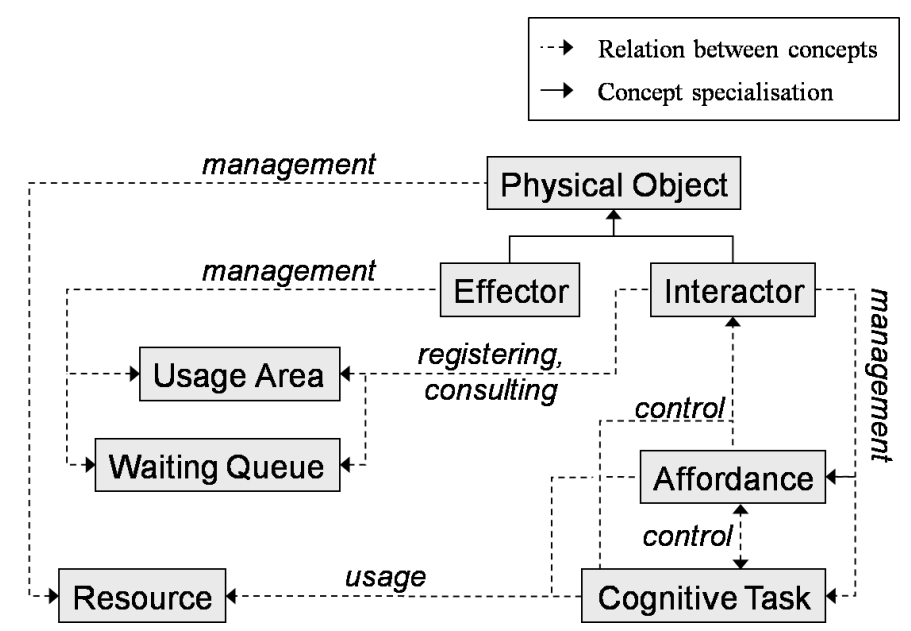

Fig. 2: Predefined set of concepts introduced with BIIO.

Physical Object: this concept describes a situated object, i.e. an object whose location is identified inside the environment. This concept is specialised in two other concepts:

Effector: this concept represents something which sustain an interaction, i.e. which participate to an interaction without having initiated it. For example, an equipment like a computer is an effector.

Interactor: this concept represents an autonomous agent which can realise an interaction. An interactor is able to manage two concepts representing its behaviours: the affordance and the cognitive task.

Affordance: this concept describes an interaction between an interactor and an effector, and so a behaviour of the interactor which is directly observable. These interactions are defined globally in the virtual environment, allowing to easily add new 
affordances without having to modify the effectors nor the interactors. An affordance represents a goal for the interactor. Thereby, the rational and cognitive layers of the interactor choose between a set of affordances requires by the interactor. Moreover, the effectors also manage the affordances they are compatible with, for example concerning the waiting queue and the usage area managements.

Cognitive Task: this concept describes an internal behaviour of the interactor, which is not directly observable. It allows to extend the internal behaviours of the agents to manage specific cases, such as simulating an agent with higher perception abilities. Some cognitive tasks are also provided for all the agent, for example to manage the primary and secondary goals in the cognitive decision layer.

Resource: this concept symbolises an internal property of a physical object, such as electricity for an equipment or the ability to move for an autonomous agent. A resource can be transitory or permanent, and can have multiple internal properties which might evolve. Both behavioural concepts (affordance and cognitive task) use resources to define their run, as for example to manage their competition. We propose four kinds of resources: basic, which can only be tested to be present or not (typically for a knowledge representation); exclusive, which can be reserved by only one process at the same time (like a member of an organism); shared, which can be reserved by several processes at the same time (like the perception ability of a human being); dynamic, which is a specialisation of the shared resource, whose parameter grows automatically (like the hunger).

Usage Area: this concept describes the positioning of an interactor in order to use an effector. There are two kinds of usage areas: single user, if the interaction with the effector is only possible for one interactor at the same time, such as getting money at a cash dispenser; multiple users, if the interaction can be performed by many interactors at the same time, such as looking at a bulletin board. Usage areas are managed by the effectors for any of their compatible affordances, and used by interactors to get informations about the way to interact.

Waiting Queue: this concept describes the way the interactors must socially organise in order to wait for a specific interaction with an effector. There are three kinds of waiting queues: none, which means that the interaction cannot be waited for; first in / first out, which means that the first interactor which is waiting for the interaction will be the next user; nearest, which means that the interactor which is the most closely with the effector will be the next user. Waiting queues are managed on the same way than usage areas.

\section{Simulation architecture}

We propose a novel crowd simulation software called SIMULEM. The architecture of this software is articulated around four phases (Figure 3), which are detailed in the following. 


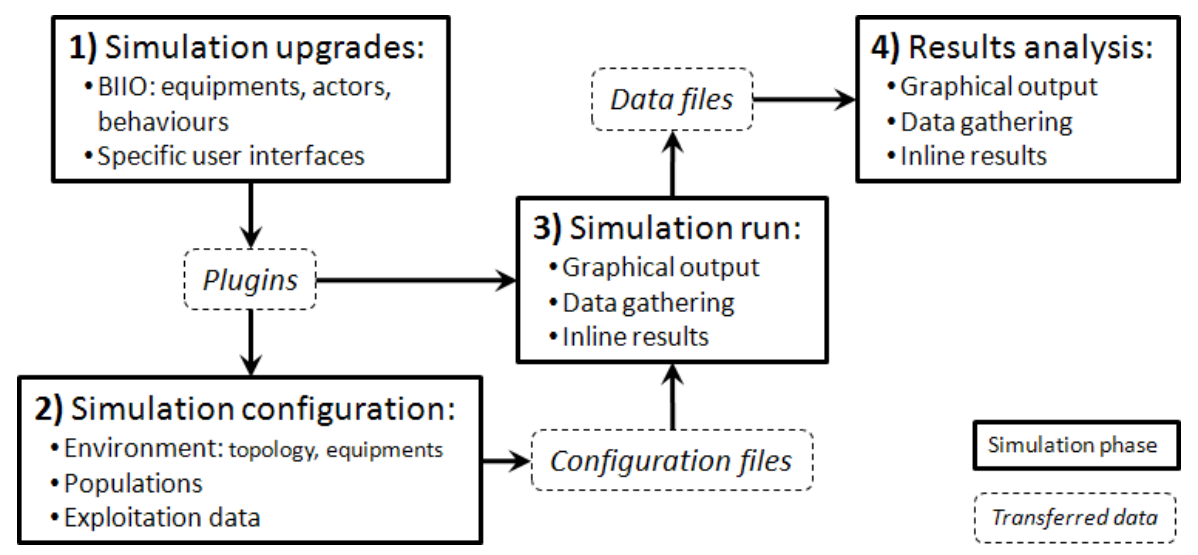

Fig. 3: The four phases of the SIMULEM's simulation architecture.

\subsection{Simulation software upgrades}

In order to easily improve the simulation tool, we propose a way to upgrade and extend its internal components thanks to external plugins. These extensions are allowed by $B I I O$, the interaction description and management framework, which allows to specialise the concepts it proposes. Additionally, some specific user interfaces can be added to the simulation software on the same principle. We will not detail these points here because they are out of the scope of this paper. One can all the same notice that this first phase is optional and is only available to computer science professionals, because of its high technical aspect. Finally, this first step produces plugins that are natively managed by the simulation software. These plugins can be used during the current simulation, and reused for any future simulation.

\subsection{Simulation configuration}

The simulation configuration phase allows the user to define information required to run a simulation. These information can be saved in an $X M L$ format, and then can be reused to run the same simulation or as source for a new simulation configuration, in order to compare many scenarii.

First, the user can define the environment of simulation by importing an external AutoCAD file (Figure 4(a)). This file format is commonly used by architects to describe the plans of buildings. We have defined modelling standards for the AutoCAD tool allowing one to indicate the topology of multi floors buildings, and to position the available equipments at the same time. Then, the simulation tool is able to automatically extract all of these data from the AutoCAD file, and to store them in a reusable $X M L$ file. From these data, the simulation tool produces an internal representation of the simulation environment thanks to a topological abstraction [11] 
which entirely preserves geometric data (Figure $4(b))$. Then, the found equipments are automatically generated inside the virtual environment and configured as defined by the AutoCAD file.

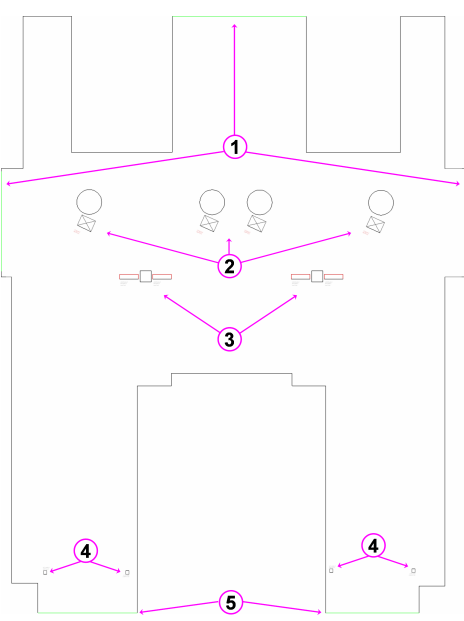

(a) Initial AutoCAD definition.

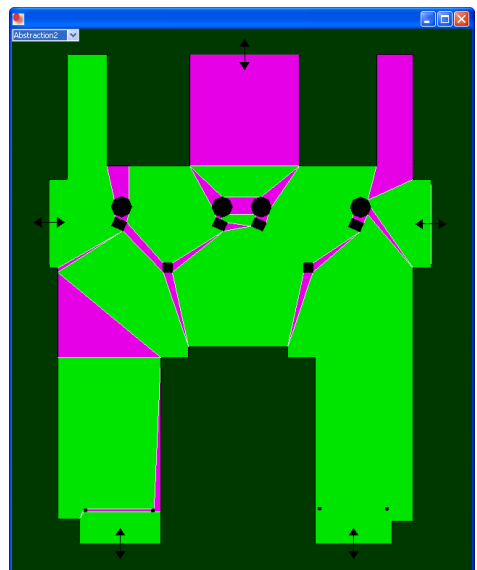

(b) Environment abstraction example.

Fig. 4: Environment configuration with SIMULEM. The numbers on the left picture represent semantical information extracted from the $\mathrm{Au}$ toCAD file: (1) standard accesses of the train station, (2) ticket selling machines, (3) departure boards, (4) ticket punchers, and (5) train accesses.

Since the virtual environment is defined, more specific configurations can be done for the populations, the trains, and the exploitation data. These configurations are directly done inside the simulation tool thanks to dedicated user interfaces. For the populations, some archetypes are first defined which corresponds to statistical distributions over the internal parameters of the simulated agents, such as speed, age, gender, size, etc. Then, populations are defined by a statistical distribution over some archetypes. For example, on figure 5) the population Population_TGVI is a distribution over the archetypes Affaire, Pendulaire, and Prive/Loisir.

Finally, the exploitation data inside a train station concern the dynamics of trains. First, a user interface allows to define some train models: the number of wagons, their capacity, the position of the doors, etc. Then, another interface contains all the data concerning the trains for the simulation (Figure 6): their unique identifier, the departure and arrival times, the destination, etc. 

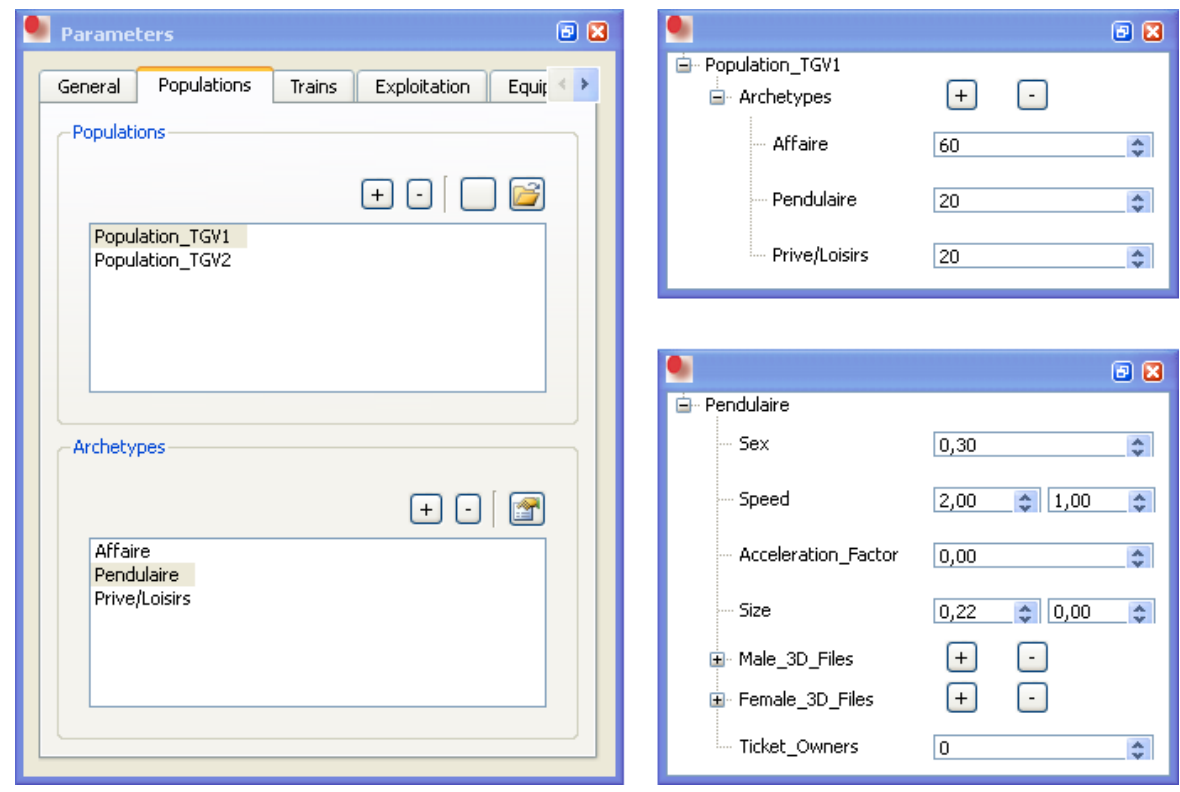

Fig. 5: Population configuration with SIMULEM. A simulated entity belongs to a population class (top right), which is a statistical distribution over archetypes (bottom right), which are each defined by a statistical distribution over the internal parameters of a humanoid.

\subsection{Simulation run}

The third phase of the software architecture consists in running the simulation. The simulation run can produce videos or pictures of the simulation. A data file is also produced, which can be saved for the fourth phase.

The simulation run can be done automatically by the tool in order to just retrieve some results for the next phase, or can allow users to interact with the simulation in order to reach two goals. First, one can change the simulation conditions inline, in order to check the impact of some events on the results. For example, a ticket selling machine could be turned off while the simulation runs, or the direction of an escalator can be changed. Second, one can extract inline results to check the evolution of the simulation. These inline results are various:

- the 3D animation of the simulation environment, with diverse camera options allowing to view all the scene (Figure 7);

- 2D maps which summarise relevant data through time: local densities of people, trajectories, or instant speeds;

- graphics which present more quantitative data: input and output flows at the accesses of the station, or the total number of people inside the environment. 


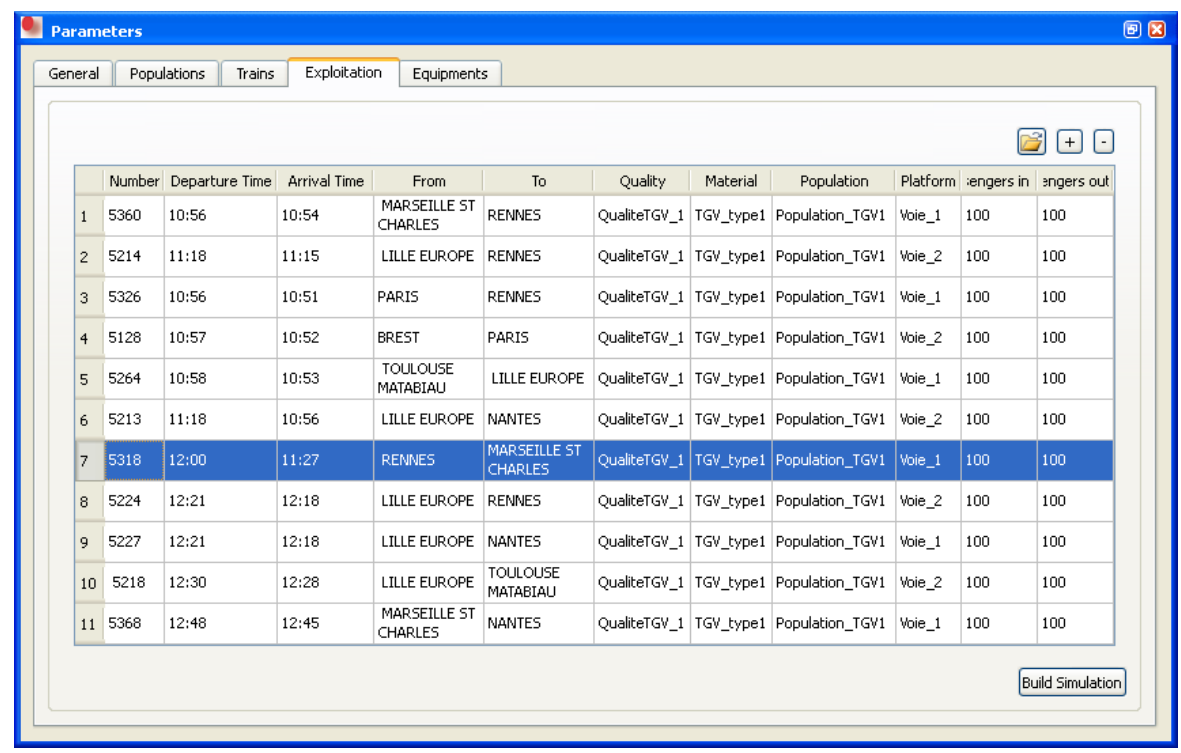

Fig. 6: Exploitation data configuration with SIMULEM. The exploitation data concerns the trains dynamics inside the station during the simulation, with all the information related to time, space, material, and involved population.

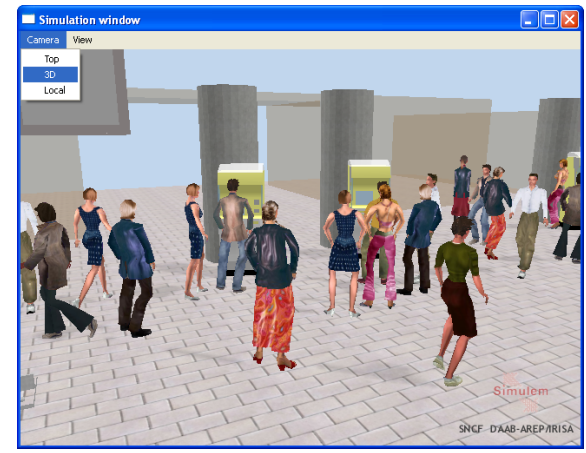

(a) .

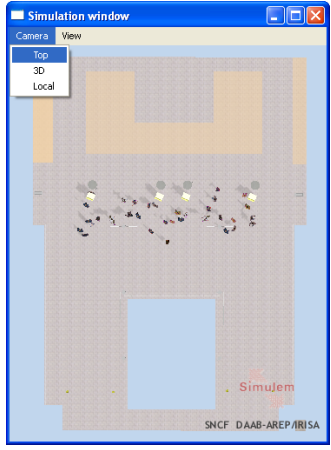

(b) .

Fig. 7: Simulation 3D output with SIMULEM.

Finally, a user can control the simulation run with a remote control. This user interface allows the standard operations: start, stop, or pause the simulation. The user can also choose the acceleration factor of the simulation time compared to real time, with a maximal value depending on the hardware performances, and a special free run value without time constraint. This tool also present the time information to user: the simulation time, and the currently reached acceleration factor. 


\subsection{Results analysis}

The fourth and last phase of the simulation consists in the offline analysis of the results of simulation runs. The analysis uses the data saved by the previous phase, which are mainly individual positions recorded over time for every simulated entities. These data can be presented to the user with graphical outputs and 2D maps, on the same way than the online results (maps and graphics are illustrated on figure 8). Moreover, the user can freely navigate through time to show the results of different instants. Finally, the user can select the time interval he wants to either show instantaneous results, or average ones over a long period.
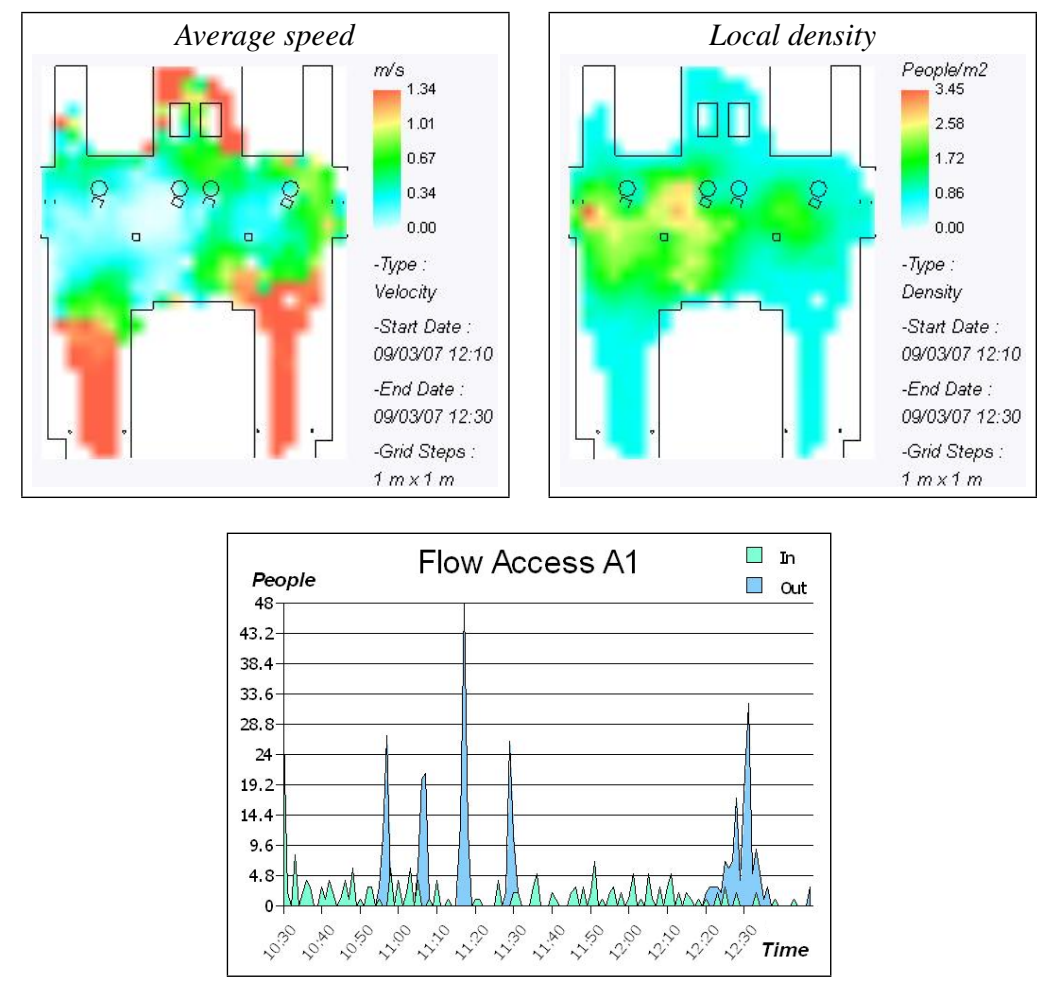

Fig. 8: Examples of the results output of SIMULEM. 2D maps: the average speeds (left) and local densities of people (right) for a given time interval in the environment. Bottom graph: incoming and outgoing flows of people for one access of the environment through time. 


\section{Results}

The SIMULEM architecture of simulation is industrially operated by our of AREP and $S N C F$ for the validation of exchange areas, and principally train stations (Figure 9). The simulation performances are very good, allowing to fully simulate approximately 2,000 agents with full behavioural abilities in real time (without graphical output which divides the performances by two). Such high results allow to perform simulations of large environments with very high populations in a reasonable time, while maintaining a good behavioural realism.
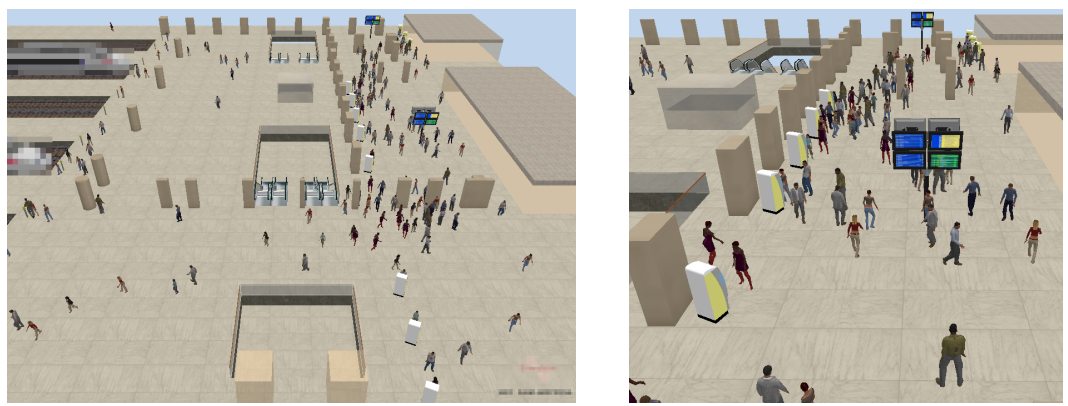

Fig. 9: Crowd simulation of a real case: Gare de Lyon in Paris. All agents are fully autonomous with individual behaviours.

\section{Conclusion and future work}

We have proposed in this paper a novel crowd simulation architecture called SIMULEM. This architecture is easily upgradable to manage new simulation cases, either concerning the environment and equipments, or the actors of the simulation. Moreover, this architecture handles all the stages of the simulation, from the environment's description, to the simulation run, the results extraction, and finally their analysis. The simulation kernel is based on the most recent approaches of cognitive agent models, integrating a goal oriented behavioural resolution. Thereby, SIMULEM does not only simulate people navigation, like actual evacuation simulators, but allows to perform a new kind of simulation where the goals of people are taken into account.

Our future work can be divided in two parts. First, we plan on upgrading the agent's model with a social layer, allowing to manage groups of people. These groups will represent static social structures, like a family, or emergent structures, like the emergent gathering of people with a similar attitude. Second, we plan on upgrading the simulation application for many purposes. We could upgrade the set of proposed $B I I O$ concepts in order to simulate new kinds of environments, by proposing new 
effectors and affordances. This perspective will also require new user interfaces depending on the kind of exploitation data of the new environments. We have also recently proposed a virtual reality application where a user can be immersed in the simulation by taking control of an agent. This new possibility allows new validation perspectives, for example to check the visibility of signs depending on the density of people. Another possible application of this improvement is to cure psychological sickness, like agoraphobia.

\section{Acknowledgement}

We wish to thank our industrial partners of AREP and SNCF for their implication in this project. We also want to thank the ANR institution to have support the SIMULEM project (reference ANR05RNTL02501). Finally, we want to thank the members of our team which have provided the $M K M[9]$ animation module.

\section{References}

1. Dirk Helbing, I. Farkas, and Tamas Vicsek. Simulating dynamical features of escape panic. Nature, 407:487-490, 2000.

2. Dirk Helbing, Lubos Buzna, and Torsten Werner. Self-organized pedestrian crowd dynamics and design solutions. In Traffic Forum 12, 2003.

3. Dirk Helbing, Lubos Buzna, Anders Johansson, and Torsten Werner. Self-organized pedestrian crowd dynamics: Experiments, simulations, and design solutions. Transportation Science, 39(1):1-24, 2005.

4. G. Keith Still. Crowd Dynamics. PhD thesis, University of Warwick, Department of Mathematics, August 2000.

5. Legion. Technical report, Legion Limited, London, United Kingdom.

6. Allen Newell. Unified theories of cognition. Harvard University Press, Cambridge, Massachusetts, 1990.

7. Sébastien Paris, Julien Pettré, and Stéphane Donikian. Pedestrian reactive navigation for crowd simulation: a predictive approach. Computer Graphics Forum, Eurographics'07, 2007.

8. Sébastien Paris, Stéphane Donikian, and Nicolas Bonvalet. Towards more realistic and efficient virtual environment description and usage. In First International Workshop on Crowd Simulation (V-Crowds'05). VRlab, EPFL, 2005.

9. R. Kulpa, F. Multon, and B. Arnaldi. Specific representation of motions for interactive animation with several characters. Computer Graphics Forum, 24(3):343-352, 2005.

10. Sébastien Paris, Stéphane Donikian, and Nicolas Bonvalet. Behavioural interactive and introspective objects for crowd simulation. In ACM SIGGRAPH / Eurographics Symposium on Computer Animation (poster session), sep 2006.

11. Sébastien Paris, Stéphane Donikian, and Nicolas Bonvalet. Towards more realistic and efficient virtual environment description and usage. In First International Workshop on Crowd Simulation (V-Crowds'05). VRlab, EPFL, nov 2005. 